đó làm cho quá trình lành vết thương được thuận lợi hơn.

\section{KẾT LUÂ̂N}

Bảo tồn không mổ là lựa chọn hàng đâu trong điều trị chấn thương thận hiện nay. Các biện pháp can thiệp ít xâm lấn như can thiệp mạch và nội soi tiết niệu có thể xử lý được các thương tổn mạch máu và đường bài tiết được phát hiện ngay từ đâu hoặc xuất hiện trong quá trình theo dõi điêuu trị, các thương tổn mà trước đây thường được chỉ định phẫu thuật. Chính vì vậy, việc áp dụng các can thiệp ít xâm lấn đã làm tăng tỷ lệ thành công của điều trị bảo tôn không mổ chấn thương thận.

\section{TÀI LIÊU THAM KHẢO}

1. Vũ Nguyễn Khải Ca (2001). Nghiên cứu chẩn đoán và điều trị phẫu thuật chấn thương thận, Luận văn Thạc sĩ Y học, Đại học Y Hà Nội, Hà Nội, $53-57$.

2. Trân Văn Sána, Trân Naoc Sinh (2011), Chấn thươna thân và vết thươna thân. Bài Giảna Bênh học niệu khoa, Nhà xuất bản Phương Đông, 9-48.

3. Bruce L.M., Croce M.A., Santaniello J.M., et al
(2001), Blunt renal artery injury: incidence, diagnosis and management, The American Journal of Surgery, 67: 550-554.

4. Fisher R.G., Ben-Menachem Y., Whigham C. (1989), Stab wounds of the renal artery branches: angiographic diagnosis and treatment by embolization, AJR Am J Roentgenol, 152 (6): 1231-5.

5. Keihani S., Anderson R.E., Fiander M., et al. (2018). Incidence of urinary extravasation and rate of ureteral stentina after hiah-arade renal trauma in adults: a meta-analvsis. Transl Androl Urol. $\quad 7$ (Suppl 2): $\quad$ S169-S178. doi:10.21037/tau.2018.04.13.

6. Miller D.C., Forauer A., Faerber G.J. (2002), Successful angioembolization of renal artery pseudoaneurysms after blunt abdominal trauma, Uroloqv, 59(3): 444.

7. Nao T.C., Lee J.J., Gonzalao M.L. (2010), Renal Dseudoaneurvsm: an overview. Nat Rev Urol. 7(11): 619-625.

8. Phillips B.J., Mirzaie M., Holzmer S., et al (2017), Penetrating Renal Trauma: A Review of Modern Management, J Eme Med Int Care, 3(3): 121.

9. Ramaswamy R.S., Darcy M.D (2016), Rterial Embolization for the Treatment of Renal Masses and Traumatic Renal Injuries, Tech Vasc Interv Radiol, 19: 203-10.

\title{
ĐĂC ĐIỂM HÌNH ẢNH CỦA 99MTc-MAA SPECT/CT TRONG LÂP KẾ HOẠCH ĐIỀU TRI UNG THƯ GAN BẰNG HẠT VI CẦU GẮN YTTRIUM-90 Ở BỆNH NHÂN UNG THƯ GAN NGUYÊN PHÁT
}

\section{TÓM TẮT}

Tổng quan: 99mTc-MAA SPECT/CT được sử dụng để đánh giá sư phân bố của hat vi câu và ước tính liều hấp thu tại khối $u$. Hơn nữa, cho đến nay, đặc điểm hình ảnh của 99mTc-MAA SPECT/CT vẫn chửa đước đánh giá một cách đầy đủ. Do vậy, chúng tôi tiển hành nghiên cứu nhằm đánh khác sự khác biệt giữa hình ảnh ${ }^{99 m T C-M A A ~ S P E C T / C T ~ s o ~ v o ̛ ́ i ~ h i ̀ n h ~ a ̉ n h ~ p l a n a r ~}$ trong lập kế hoạch điêu trị. Đối tượng và phương pháp: 52 bệnh nhân ung thư gan nguyên phát, giai đoạn trung gian và tiến triển được điêu trị tắc mạch xạ trị bằng hạt vi cầu gắn ${ }^{90}$ Y (TARE) được chọn vào nghiển cứu. Đặc điểm hình ảnh và các thông số lập kế hoach điều trị: shunt gan - phổi (LSF\%), chỉ số giữa khối u và gan lành ( $\mathrm{TNr}$ ) và liều hấp thụ của khối u (Dtumor) được thực hiên và đối chiếu trên ${ }^{99 \mathrm{~m} T \mathrm{~T}-\mathrm{MAA}}$ SPECT/CT và planar. Kết quả: Các đặc điểm hình ảnh cân đánh giá gồm: mật độ phân bố phóng xạ, hoại tử

*Bệnh viện TUQQĐ 108

Chiu trách nhiệm chính: Mai Hồng Sơn

Email: alex.hong.son@gmail.com

Ngày nhận bài: 17.12.2020

Ngày phản biện khoa họ: 21.01.2021

Ngày duyệt bài: 2.2.2021
Mai Hồng Sơn*, Nguyễn Bình An*, Lê Ngọc Hà*

và huyết khối được quan sát trên SPECT/CT tốt hơn so với planar. Tướng quan và đồng thuận của $\mathrm{TNr}$ trên SPECT/CT và planar ở mức trung bình, sự khác biệt có ý nghĩa thống kê $(P<0,05)$. Liêu chiếu khối u trển planar là 120 nhỏ hơn so với 155 trên SPECT/CT $(P<0,05)$. Kết luân: ${ }^{99 m T C-M A A ~ S P E C T / C T ~ p h a ́ t ~ h i e ̣ ̂ n ~}$ được đăc điểm hình ảnh khối $u$, huyết khối tĩnh mach cửa và ước tính liều điều trị cao hơn so với planar.

Tư khóa: Ung thư biểu mô tế bào gan, lập kê hoạch điều trị, tắc mạch xạ trị, hạt vi cầu gắn ${ }^{90} Y$, 99mic-MAA SPECT/CT, planar.

\section{SUMMARY}

\section{CHARACTERISTIC IMAGING OF 99M TC-MAA SPECT/CT IN PRE-TREATMENT PLANNING OF ${ }^{90} Y$ RESIN MICROSPHERES IN HEPATOCELLULAR CARCINOMA}

Background and aim: 99mTC-MAA SPECT/CT imaging is performed after radioembolization to reevaluate the resin microsphere distribution and estimate the absorbed radiation dose of target tumor. However, role of 99mTc-MAA SPECT/CT simulation was not well established until now. The purpose of our study is to validate the utility of SPECT/CT compared to planar simulation in dosimetry. Material and method: Fifty - two consecutive HCC patients, 
intermediate and advanced stage who underwent ${ }^{90} \mathrm{Y}$ resin microsphere transarterial embolization (TARE) were recruited in the study. Image characteristic and parameters: lung shunt fraction (LSF), tumor tonormal liver uptake ratio (TNr) and absorbed dose for target tumors were estimated on 99mTc-MAA SPECT/CT and planar. Results: The imaging characteristics including heterogeneity, necrosis and thrombosis uptake were better delineated on SPECT/CT imaging than planar. The agreement and correlation of $\mathrm{TNr}$ on SPECT/CT and planar were average $(p<0,05$. Dtumor on planar is lower than that on SPECT/CT with significant difference $(P<0,05)$. Conclusions: ${ }^{99 m T C-M A A ~ S P E C T / C T ~ i s ~ s u p e r i o r ~ t o ~}$ planar in simulation before treatment ${ }^{90} \mathrm{Y}$ resin microsphere in tumor delineantion and dosimetry.

\section{I. ĐĂT VẤN ĐỀ}

Ung thư biểu mô tế bào gan (UBTG) là một trong những bệnh ung thư gây tử vong hang đầu ở khu vực Châu Á Thái Bình Dương (1). Hạt vi cầu gắn ${ }^{90} Y$ là một trong những phương pháp điều trị hứa hẹn mang lại hiệu quả cho bệnh nhân ung thư gan nguyên phát (2).

Trong thực hành lâm sàng, tắc mạch xạ trị (RE) sử dụng hạt vi cầu gắn ${ }^{90} Y$ định hướng mồ phỏng trên xạ hình tưới máu động mạch bằng 99mTc-macro aggregated albumin ( ${ }^{99 \mathrm{~m}} \mathrm{Tc}-\mathrm{MAA}$ ). Mục đích của kĩ thuật này là đạt liều chiếu tối đa tại $u$ và tối thiểu tại tổ chức gan lành. Chỉ số shunt gan - phổi và chỉ số giữa khối u và gan lành trên xạ hình ${ }^{99 \mathrm{~m} T c-M A A}$ trong lập kế hoạch điều trị là mấu chốt để tối đa liều chiếu tối đa tại u và tối thiểu tại gan lành và phổi. Phương pháp tính liều từng phần sử dung hình planar ${ }^{99 \mathrm{~m} T \mathrm{~T}-}$ MAA đã được sử dụng để ước tính liều hấp thụ tại u trước khi điều trị hat vi cầu gắng ${ }^{90} Y$. Hiện nay, ${ }^{99 m T C-M A A ~ S P E C T / C T ~ đ u ̛ o ̛ ̣ c ~ k h u y e ̂ ́ n ~ c a ́ o ~ s u ̛ ̉ ~}$ dụng để mô phỏng phân bố hạt vi câuu gắn ${ }^{90}$ Y. 99mTC-MAA SPECT/CT sau điều trị cần được đối chiếu với planar thông thường. Do đó, đối chiếu giá trị mô phỏng planar với ${ }^{99 m}$ Tc-MAA SPECT/CT cần được làm rõ.

Các nghiên cứu khác cho thấy ${ }^{99 m} T c-M A A$ SPECT/CT tương quan tốt với planar trong đánh giá chỉ số giữa khối u và gan lành có giá trị phát hiện các vị trí bắt xạ ngoài gan để phân tâng tổn thương các cơ quan lành do xạ trị (3). Hiện nay, chưa nhiêu nghiên cứu so sánh đặc điểm hình

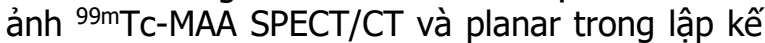
hoạch điều trị ở bệnh nhân HCC.

Nghiên cứu tiến hành đánh giá đặc điểm hình ảnh và các thông số lập kễ hoạc điều trị trên 99mTc-MAA SPECT/CT và so sánh với planar trong tiên lượng đáp ứng điều trị ở bệnh nhân điều trị hạt vi cầu gắn ${ }^{90} \mathrm{Y}$.

\section{II. ĐỐI TƯỢNG VÀ PHƯƠNG PHÁP NGHIÊN CỨU}

Bênh nhân: 52 bênh nhân ung thư gan nguyển phát, giai đoạn trung gian và tiến triển được điều trị tắc mạch xạ trị bằng hạt vi cầu gắn ${ }^{90}$ Y (TARE) từ 5/2017 đến 12/2020 được chọn vào nghiên cứu. Bệnh nhân được chọn vào nghiên cứu có chức năng gan bảo tồn (ChildPugh $A$ ) và ECOG từ $1-2$. Chỉ định điều trị TARE được xem xét và quyết định bởi tiểu ban ung thư gan - bệnh viện 108. Các bệnh nhân đồng ý tham gia vào nghiên cứu. Bệnh nhân có huyết khối nhánh chính tĩnh mạch cửa, ECOG > 2, xơ gan Child-Pugh $C$ được loai khỏi nghiên cứu.

Mô phỏng, điêu trị và đánh giá đáp ứng: Chụp CT đa dãy cản quang vùng bụng đánh giá bản đồ mạch, ước tính thể tích gan lành và thể tích u điều trị. Chụp mạch chẩn đoán mô phỏng để xác định bản đồ động mạch và vị trí đặt catheter tối ưu để điêu trị TARE. Tiêm $5 \mathrm{mCi}$ 99mTc-MAA vào động mạch nuôi khối u gan. Trong trường hợp có nhiều $u$ trên cùng một phân thùy hoặc được cấp máu nhiều hơn 2 nhánh động mạch gan thì tiêm ${ }^{99 m T c-M A A ~ v a ̀ o ~}$ các nhánh động mạch phù hợp là các nhánh động mạch đích điêu trị.

Bênhh nhân chụp xạ hình SPECT/CT và planar trên hệ thống gamma camera 2 đâu thu (Optima 610, GE Healthcare, Milwaukee, WI, USA) tại khoa y học hạt nhân, bệnh viện 108 sau khi tiêm 99mTc-MAA 1 giờ. Trường chụp hình cần có chứa cả phổi và bụng. Sử dụng collimators năng lượng thấp, độ phân giải cao và cài đặt cửa sổ năng lượng $140 \pm 10 \mathrm{KeV}$. Hình SPECT chụp 90 điểm dừng, shoot mode, kích thước ma trận $256 \mathrm{x}$ 256. Chụp CT sau SPECT có điện thế $120 \mathrm{kV}$, dòng qua ống tia $30 \mathrm{~mA}$ và độ dày lát cắt $5 \mathrm{~mm}$.

Hình chụp được trình bày trên hệ thống Xeleris 4.0 (GE Healthcare, Milwaukee, WI, USA) trên các bình diện axial, sagittal và coronal. Vẽ vùng quan tâm (ROIs) ở phổi, toàn bộ gan và khối u cần điều trị ở hình planar. Sử dụng phần mềm Dosimetry toolkit (GE Healthcare, Milwaukee, WI, USA) vẽ ROIs ở phổi, gan và u ở từng lát cắt một trên cả SPECT/CT và planar. Vùng u hoại tử được loại trừ. Ước tính chỉ số shunt gan - phổi (LSF\%) và chỉ số giữa khối u và gan lành (TNr) dựa vào số đếm phóng xa ở mỗi ROI trên cả planar và SPECT/CT theo công thức trong hướng dẫn của EANM (4). Đối với u có nhiều nguồn động mach thì TNr xác định cho từng u. Ước tính liều hấp thụ trung bình ở phổi (Dlung), gan lành (Dliver) và tổ chức u (Dtumor) bằng phương pháp tính liều từng phần. Liều hấp thụ tại u nên > 120Gy, Dlung < 20Gy và Dliver 
< 30Gy (5). Trường hô nhiều u nằm trong cùng phân thùy hoặc nhiêu hơn 2 động mạch gan tiến hành điều trị siêu chọn lọc dựa trên phương pháp tính liều từng phần. Dliver và Dtumor được ước tính chọn lọc cho từng u và tổ chức gan lành.

Sau khi tính liều điều trị, liều hạt vi cầu gắn 90Y (SIR-Sphere, SIRTeX ${ }^{\mathrm{TM}}$, Sydney, Australia) tương ứng được tiêm vào động mạch gan cấp máu cho u. Bệnh nhân được chụp PET/CT tại khoa y học hạt nhân sau điêu trị 6 giờ. Quy trình chụp được mô tả ở nghiên cứu trước đó (6). Kết quả hình ảnh gồm: đặc điểm bắt xạ của $u$, hoại tử và bắt xạ của huyết khối trên SPECT/CT và planar sau điều trị được phân tích riêng biệt và thống nhất bởi 2 bác sĩ y học hạt nhân có kinh nghiệm. Vẽ ROIs ở phổi, gan và u để ước tính LFS\% và TNr. So sánh giá trị TNr trên hình ảnh SPECT/CT và planar.

Phân tích thống kê: Phân tích dữ liệu bằng Phần mềm thống kê (SPSS) 20.0 and GraphPad Prism (version 8.0, GraphPad software). Sử dung hệ số tương quan Pearson's và biểu đồ Bland Altman 2 trục ngẫu nhiên để đánh giá tương quan và đồng thuận các giá trị TNr. Sử dụng Chi-squared test hoăc Fisher's exact test để so sánh các biến phân loai. Sử dụng T-test bắt cặp hoăcc ANOVA lặp lại để so sánh các biến liên tục phẩn phối chuẩn và Wilcoxon signed-rank test hoặc Friedman test đối với các biến không phân phối chuẩn. Kiểm định có ý nghĩa khi $\mathrm{P} \leq 0.05$.

\section{KẾT QUẢ NGHIÊN CỨU}

Lập kế hoạch điều trị được thực hiện cho 52 bệnh nhân (66 tổn thương đích) (Bảng 1). Khoảng 14/52 (27\%) bệnh nhân được lập kế hoạch cho 2 nguồn động mạch nuôi khối u. Trong nghiên cứu của chúng tôi, u thường gặp ở gan phải nhiều hơn gan trái (64/66 so với $2 / 66$ bệnh nhân). Thể tích trung bình của khối u là $479,57 \pm 379,14$ ml, 52,9\% bệnh nhân có huyết khối và $35 \%$ có u hoaai tử.

Bảng 1. Đặc điểm chung của bệnh nhân $(n=52)$

\begin{tabular}{|c|c|}
\hline Lâm sàng & Giá tri \\
\hline Tuổi (số tuối, tuối trung bình \pm SD) & $56.25 \pm 14,15$ \\
\hline \multicolumn{2}{|l|}{ Giới } \\
\hline Nam & $49(94.2 \%)$ \\
\hline Nữ & $3(5,8 \%)$ \\
\hline \multicolumn{2}{|l|}{ Bênh gan kết hợp } \\
\hline Viêm gan virus $B$ & $53(97 \%)$ \\
\hline Viêm gan virus $C$ & - \\
\hline \multicolumn{2}{|l|}{ Vị trí u } \\
\hline Gan phải & $(94,2 \%)$ \\
\hline Gan trái & $(5,8 \%)$ \\
\hline Thể tích u (ml, trung bình \pm SD) & $\begin{array}{c}479,57 \pm \\
379,14\end{array}$ \\
\hline Huyết khối nhánh tĩnh mạch cửa & $52.9 \%)$ \\
\hline Hoại tử u & $35.3 \%$ \\
\hline \multicolumn{2}{|l|}{ Giai đoạn Barcelona } \\
\hline Trung gian & $17.6 \%$ \\
\hline Tiến triển & $82.4 \%$ \\
\hline Số lượng tốn thương đích & 66 \\
\hline U có 1 nhánh mạch nuôi & $67.6 \%$ \\
\hline U có 2 nhánh mạch nuôi & $32.4 \%$ \\
\hline
\end{tabular}

So sánh đặc điếm hình ảnh giữa ${ }^{99 m} T c-$ MAA SPECT/CT và planar (bảng 2). Hình ảnh SPECT/CT phát hiện được nhiều trường hợp phân bố phóng xạ không đều tại khối u hơn so với planar $(73,1 \%$ so với $50 \%$; $p<0,001$. Khối u có hoại tử được phát hiện với tỷ lệ $57,7 \%$ trên SPECT/CT cao hơn rõ rệt so với tỷ lê $13,5 \%$ trên hình ảnh planar $(p=0,015)$. Đặc biệt, hình ảnh cắt lớp SPECT/CT phát hiện được $9,6 \%$ bệnh nhân có huyết khối tăng hoạt tính phóng xạ trong khi đó không phát hiện được trên hình ảnh planar.

Bảng 2. So sánh đặc điểm hình ảnh khôi u trên ${ }^{99 m}$ TC- MAA SPECT/CT và planar

\begin{tabular}{|c|c|c|c|c|}
\hline \multirow{2}{*}{\multicolumn{2}{|c|}{ Đặc điểm hình ảnh }} & \multicolumn{3}{|c|}{ Hình ảnh ${ }^{99 m}$ Tc-MAA $(n=52)$} \\
\hline & & \multirow{2}{*}{$\frac{\text { SPECT /CT n (\%) }}{14(26,9)}$} & \multirow{3}{*}{$\begin{array}{c}\text { Planar n (\%) } \\
26(50) \\
26(50)\end{array}$} & \multirow{3}{*}{$\begin{array}{c}\mathbf{P} \\
<0,001\end{array}$} \\
\hline \multirow{2}{*}{ HPTX } & Đều & & & \\
\hline & Không đều & $38(73,1)$ & & \\
\hline \multirow{2}{*}{ Hoại tử } & Có & $30(57,7)$ & $7(13,5)$ & \multirow{2}{*}{0,015} \\
\hline & Không & $22(42,3)$ & $45(86,5)$ & \\
\hline \multirow{2}{*}{$\begin{array}{l}\text { Huyết khối } \\
\text { TMC }\end{array}$} & Có & $5(9,6)$ & $52(400$ & \multirow[b]{2}{*}{ - } \\
\hline & Không & $47(90,4)$ & $52(100)$ & \\
\hline
\end{tabular}

HTPX: hoạt tính phóng xạ, TMC: tĩnh mạch cửa

Shunt gan - phổi (\%) từ 0,8 đến 33,2 trên hình ảnh planar và từ 0,5 đến 35,8 trên hình ảnh cắt lớp SPECT/CT. Trung vị của shunt gan phổi trên hình ảnh planar nhỏ hơn trên SPECT/CT $(4,9$ so với 5,2$)$ với sự khác biệt chưa có ý nghĩa thống kê $(p=0,65)(A)$.Chỉ số TNr dao động tù̀ $0,6-19,2$ trên SPECT/CT và từ $0,5-35,8$ trên hình ảnh planar. Trung vị của TNr SPECT/CT cao hơn rõ rệt so với $\mathrm{TNr}$ planar $(5,7$ so với 3,8$)$ với $p=0,0005$ (B). Dtumor trên SPECT/CT giao động 
từ 50 - 710 Gy so với ước tính trên planar là 20

154,5 cao hơn rõ rệt so với hình ảnh planar với - 225. Trung vị của Dtumor trên SPECT/CT là sự khác biệt có ý nghĩa thống kê $(p<0,0001)$ (C).
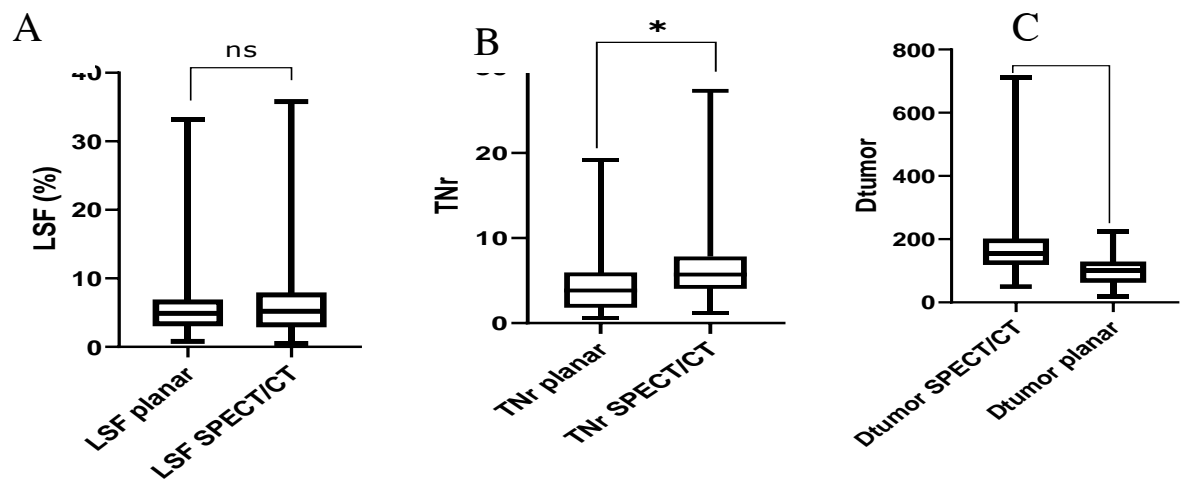

Biểu đồ 1. So sánh các thông số lập kế hoạch điều trị trên planar và SPECT/CT

\section{BÀN LUÂN}

Kết quả quan trọng đầu tiên trong nghiên cứu này là ${ }^{99 \mathrm{~m} T c-M A A}$ SPECT/CT tương đồng với

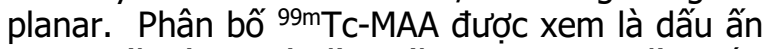
thay thế đánh giá liêu hấp thu hạt vi cầu gắn 90Y. Nhìn chung, xạ hình planar hoặc SPECT được sử dụng rộng rãi trước RE. Xạ hình ${ }^{99 m} T c-$ MAA giúp lập bản đồ mạch nuôi khối u và phát hiện các vị trí bắt xạ ngoài gan nhằm phòng ngừa biến chứng do trào ngược hạt vi cầu gắn 90Y. Một nghiên cứu khác cho thấy ${ }^{99 \mathrm{mT}} \mathrm{Tc}-\mathrm{MAA}$ SPECT/CT rất có ý nghĩa phát hiện vị trí bắt xạ ở đường tiêu hóa ngoài gan (7). Trong nghiên cứu này, chúng tôi sử dụng 99mTc-MAA SPECT/CT hướng dẫn can thiệp động mạch chọn lọc ở 10/34 bệnh nhân. Phương pháp tính liều từng phần trên SPECT/CT giúp đạt liêu hấp thụ cao tại u và liều chiếu thấp ở mô gan lành khi tắc mạch bằng hạt vi cầu gắn Y90. Hơn nữa, xác định chính xác thể tích u điều trị (loại bỏ vùng hoại tử) và phát hiện bắt xạ ngoài gan cung cấp cớ sở tính liều tai khối u. SPECT đơn thuần không thể xác định chính xác thể tích quan tâm (VOI) và ước tính thấp các thông số cần thiết(8). 99mTc-MAA SPECT/CT ước tính khối lượng u và tổ chức gan lành tốt hơn so với SPECT và hoăc planar. Trong nghiên cứu của chúng tôi, SPECT/CT phát hiện u bắt xạ không đồng nhất và hoại tử cao hơn so với hình planar.

Kĩ thuật định liều trên SPECT/CT vai trò mô phỏng định lượng quan trọng để lập kế hoạch điều trị. Theo phương pháp tính liều từng phần, chỉ số u - gan lành ( $\mathrm{TNr}$ ) biểu hiện khoảng liều an toàn khi truyền hạt vi cầu gắn $90 Y$. Ngưỡng giới hạn TNr và LSF\% là cơ sở lập kế hoạch điều trị hiệu quả. Căn cứ các chỉ số $T N r$ và $L S F \%$, ta có thể xác định hoạt độ hạt vi cầu gắn $90 Y$ cần thiết đế đạt liều chiếu tối đa ở u và giảm đáng kể liều chiểu này ở mô gan lành. Đặc biệt, khi có nhiều khối u thì SPECT/CT giúp xác định chính xác ROI của từng đích u điều trị. TNr $<2$ (tính trên ${ }^{99 m}$ Tc-MAA planar) gợi ý tình trạng không an toàn khi điều trị hạt vi cầu gắn Y-90. SPECT/CT giúp xác định chỉ số này chính xác hơn planar. Khi ước tính liều hấp thụ tiêu diệt u bằng phương pháp tính từng phần, ta có thể ước tính TNr trung bình ở từng bệnh nhân và TNr cho mối u.

Chúng tôi cũng không thể đánh giá vai trò các thông số trên SPECT/CT trong tiên đoán thời gian sống toàn bộ và thời gian sống bệnh không tiến triển ở bệnh nhân HCC không thể phẫu thuật. Bên cạnh đó, chúng tôi chưa đánh giá được mối liên quan giữa TNr, LSF và Dtumor với đặc đặc điểm bắt xạ, kích thước u và huyết khối tĩnh mạch cửa. Số lượng bệnh nhân ở mối nhóm quá ít nên không thể tiến hành phân tích thống kê. Nên sử dụng công cụ mô phỏng Kernel hoặc Montecarlo để ước tính mức độ đồng nhất của liều hấp thụ trên từng phần của u. Chúng tôi chỉ sử dụng phương pháp tính liều từng phần (partion model) để mô phỏng có thể không đánh giá chính xác hoàn toàn liều hấp thụ tại u.

\section{KẾT LUÂ̂N}

Nghiên cứu cho thấy ${ }^{99 m T C-M A A ~ S P E C T / C T ~ l a ̀ ~}$ công cụ mô phỏng đáng tin cậy khi đối chiếu với planar. Cần tiếp tục nghiên cứu để đánh giá vai trò của xạ hình SPECT/CT trong tiên lượng đáp ứng điều trị và thời gian sống thêm toàn bộ.

\section{TÀI LIẸU THAM KHẢO}

1. Zhu RX, Seto W-K, Lai C-L, Yuen M-F. Epidemiology of Hepatocellular Carcinoma in the Asia-Pacific Region. Gut and liver. 2016;10(3):332-9.

2. Salem R, Lewandowski RJ, Mulcahy MF, Riaz A, Ryu RK, Ibrahim S, et al. Radioembolization 
for hepatocellular carcinoma using Yttrium-90 microspheres: a comprehensive report of long-term outcomes. Gastroenterology. 2010;138 (1): 52-64.

3. Ahmadzadehfar $H$, Sabet $A$, Biermann $K$, Muckle M, Brockmann $\mathrm{H}$, Kuhl $\mathrm{C}$, et al. The significance of 99mTc-MAA SPECT/CT liver perfusion imaging in treatment planning for $90 \mathrm{Y}$ microsphere selective internal radiation treatment. Journal of nuclear medicine : official publication, Society of Nuclear Medicine. 2010;51(8):1206-12.

4. Giammarile F, Bodei L, Chiesa C, Flux G, Forrer $\mathbf{F}$, Kraeber-Bodere $\mathbf{F}$, et al. EANM procedure guideline for the treatment of liver cancer and liver metastases with intra-arterial radioactive compounds. European journal of nuclear medicine and molecular imaging. 2011;38(7):1393-406.

5. Gil-Alzugaray $B$, Chopitea $A$, Iñarrairaegui $M$, Bilbao JI, Rodriguez-Fraile M, Rodriguez J, et al. Prognostic factors and prevention of radioembolization-induced liver disease. Hepatology (Baltimore, Md). 2013;57(3):1078-87.
6. Gates VL, Esmail AA, Marshall K, Spies S, Salem R. Internal pair production of $90 Y$ permits hepatic localization of microspheres using routine PET: proof of concept. Journal of nuclear medicine : official publication, Society of Nuclear Medicine. 2011;52(1):72-6.

7. Lenoir L, Edeline J, Rolland $Y$, Pracht $M$, Raoul J-L, Ardisson V, et al. Usefulness and pitfalls of MAA SPECT/CT in identifying digestive extrahepatic uptake when planning liver radioembolization. European journal of nuclear medicine and molecular imaging. 2012;39(5):872-80.

8. Garin $E$, Lenoir L, Rolland $Y$, Laffont $S$, Pracht $M$, Mesbah $H$, et al. Effectiveness of quantitative MAA SPECT/CT for the definition of vascularized hepatic volume and dosimetric approach: phantom validation and clinical preliminary results in patients with complex hepatic vascularization treated with yttrium-90labeled microspheres. Nuclear medicine communications. 2011;32(12):1245-55.

\section{NGHIÊN CỨU CHU TRÌNH ĐÀO TẠO (LEARNING CURVE) PHẪU THUÂT RÔ BỐT DA VINCI XI ĐIỀU TRI UNG THƯ ĐẠI TRỰC TRÀNG TẠI BỆNH VIÊ̂N K}

\section{TÓM TẮT}

Mục tiêu: Đánh giá kết quả chu trình đào tạo phẫu thuât rô bốt Da Vinci Xi điều tri ung thư đai trực tràng tại bệnh viện K. Phương pháp nghiên cứu: mô tả tiền cứu. Kết quả: 31 bệnh nhân, 21 nam -10 nữ, Tuổi trung bình 56,7; vị trí ung thư: trực tràng thấp 2 , trực tràng trung bình 6 , trực tràng cao 14 , đại tràng sigma 5 , đại tràng phải 4 ; Phương pháp phấu thuật: cắt cụt trực tràng 2 , cắt đoạn trực tràng 20 , cắt đại tràng sigma 5 , cắt đại tràng phải 4.Thời gian mổ trung bình 139,5 \pm 25 ,8phút, số lượng hạch nạo vét

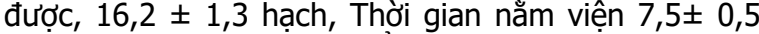
ngày, biến chứng sau mổ $1(4,5 \%)$ độ 1 Clavien, chuyển mổ mở $0 \%$, diện cắt dưới u $100 \%$ không còn tế bào ung thư. Kết luận: Chu trình đào tao mổ rô bốt Da Vinci Xi ung thư đại trực tràng đạt kết quả tốt qua 31 trường hợp phẫu thuật.

Tư khóa: Chu trình đào tạo rô bốt, ung thư đai trực tràng

\section{SUMMARY}

STUDY OF LEARNING CURVE IN ROBOTIC SURGERY FOR COLORECTAL CANCER TREATMENT BY DA VINCI XI SYSTEM AT K HOSPITAL

Purpose: Evaluating the result of learning curve in

\section{*Bênh viên $K$}

Chịu trách nhiệm chính: Phạm Văn Bình

Email: binhva@yahoo.fr

Ngày nhận bài: 21.12 .2020

Ngày phản biên khoa hoc: 25.01.2021

Ngày duyệt bài: 8.2 .2021 robotic surqery treatment colorectal cancer at $\mathrm{K}$ hospital by da Vinci Xi system. Method: prospective and descriptive study. Result: 31 patients, 21 males and 10 females. The averaqe of aqe was 56.7. Location of tumor: lower rectal (2patients), upper rectal (14 patients), siqmoid colon (5 patients), riqht colon ( 4 patients). Type of surqery: abdominoperineal resection ( 2 patients), low anterior resection (20 patients), right colectomy (4 patients). Time of operation was $139.5 \pm 25.8$ minutes. Lenqth of hospitalization was $7.5 \pm 0.5$ day. Postoperative complications: 1 patient (4.5\%) - qrade I of Clavien, conversion surqery none case. $31 / 31$ patients had distal marqin free. Conclusion: The learning curve in robotic surqery for colorectal cancer treatment by daVinci Xi has been successful with 31 patients,

Key words: learning curve in robotic surgery, colorectal cancer.

\section{I. ĐĂT VẤN ĐỀ}

Phẫu thuật sang chấn tối thiểu trong ung thư đại trực tràng (UTĐTT) đã được chứng minh là an toàn và khả thi. Lợi ích của phẫu thuật xâm lấn tối thiểu mang lại khi mổ UTĐTT như giảm đau sau mổ, hồi phục nhanh, ít biến chứng tắc ruột , viêm phổi, sa lồi thành bụng... là những ưu điểm đã được thừa nhận. Những nghiên cứu gần đây cũng đã chứng minh kết quả về măt ung thư học của phẫu thuật xâm lấn tối thiểu tương đướng với mổ mở [2],[3],[5]. Sự phát triển về kỹ thuật ngoại khoa nhờ vào những tiến 